\title{
Correlation of Histopathologic Findings of Non-Graft Threatening Preservation/Reperfusion Injury in Time-Zero Liver Needle Biopsies With Short-Term Post-transplantation Laboratory Alterations
}

\author{
Narges Shahbazi ${ }^{1}$; Hayedeh Haeri ${ }^{1}$; Mohsen Nasiri Toosi ${ }^{2,3}$; Ali Jafarian ${ }^{3,4}$; Reza Shahsiah ${ }^{1,3}$; \\ Monavar Talebian Moghadam ${ }^{3}$; Sedigheh S. Poursaleh ${ }^{5}$; Farid Azmoudeh-Ardalan ${ }^{1,3,}$ \\ ${ }_{1}^{1}$ Department of Pathology and Laboratory Medicine, Cancer Institute, Imam Khomeini Hospital Complex, Tehran University of Medical Sciences, Tehran, IR Iran \\ ${ }_{3}^{2}$ Department of Internal Medicine, Gastroenterology and Hepatology Division, Imam Khomeini Hospital Complex, Tehran University of Medical Sciences, Tehran, IR Iran \\ ${ }^{3}$ Liver Transplantation Research Center, Tehran University of Medical Sciences, Tehran, IR Iran \\ 4 Department of General Surgery, Hepatobiliary and Liver Transplantation Division, Imam Khomeini Hospital Complex, Tehran University of Medical Sciences, Tehran, IR Iran \\ 5 Iranian Tissue Bank and Research Center, Imam Khomeini Hospital Complex, Tehran University of Medical Sciences, Tehran, IR Iran \\ ${ }^{*}$ Corresponding Author: Farid Azmoudeh-Ardalan, Department of Pathology and Laboratory Medicine, Imam Khomeini Hospital Complex, Tehran University of Medical Sciences, P. \\ O. Box: 14197-33141, Tehran, IR Iran. Tel: +98-9121055232, Fax: +98-2188953010, E-mail: azmoudeh@sina.tums.ac.ir
}

Received: May 16, 2015; Accepted: June 10, 2015

\begin{abstract}
Background: Early post-transplantation alterations in liver tests are caused by a variety of etiologies including rejection, biliary or vascular complications, and preservation/reperfusion injury (PRI).

Objectives: The aim of this study was to show the correlation between histopathologic changes of PRI and the alterations in liver tests in the early post-transplantation period.

Materials and Methods: Between April 2013 and August 2014, histopathologic findings of protocol, time-zero, Tru-Cut, liver needle biopsies were evaluated in 94 cases of cadaveric liver transplantation. The histopathologic changes included ballooning degeneration, micro- and macro-vesicular steatosis, bilirubinostasis, apoptotic cells, bile plugs and neutrophilic infiltration. These histopathologic changes were compared with the early (15 days) post-transplantation liver laboratory findings.

Results: Clinico-pathologic evaluation of all 94 cases was done by assessment of PRI findings in time-zero biopsies and possible causes of allograft injury were appraised. In 21 patients, a specific cause for allograft injury was found including rejection and/or surgical complications. In the remaining 73 cases, there was no specific cause for allograft injury and histopathologic findings of time-zero liver needle biopsies supported PRI. We classified liver laboratory tests alterations as: hepatocellular damage (elevation of transaminases and lactate dehydrogenase), cholestatic damage (elevation of alkaline phosphatase and total bilirubin) and mixed. Hepatocellular and cholestatic alterations in liver function tests were associated with the presence of marked apoptotic bodies and neutrophilic aggregates in time zero biopsies, respectively. On the other hand, macrovesicular steatosis was dominantly associated with mixed (hepatocellular and cholestatic) laboratory alterations of liver tests.

Conclusions: Any discrepancy between histopathologic changes in time-zero biopsies and pattern of early liver laboratory alterations may be considered as a warning for causes other than PRI.
\end{abstract}

Keywords: Ischemia Reperfusion Injury; Liver Function Tests; Pathology; Liver Transplantation

\section{Background}

Preservation/reperfusion injury (PRI) is an inherent complication of liver transplantation and because of increase in selection of marginal organs, this injury is burgeoning. Warm ischemia mainly affects hepatocytes and leads to hepatocyte apoptosis $(1,2)$. On the other hand, cold ischemia targets nonparenchymal cells in the allograft, causing sinusoidal endothelial cell apoptosis probably by caspasedependent pathways or changes in intracellular $\mathrm{pH}(2,3)$. Adhesion of neutrophils to the site of endothelial damage and imbalance in vasodilator/vasoconstrictor cytokines can cause microcirculatory disturbances and further cellular injury (4-7). Bile duct damage has also been reported as a consequence of cold ischemia (8).
After reconstitution of blood supply, hepatocyte injury becomes clinically apparent by elevated transaminases and also hyperbilirubinemia when injury is severe (9). Non-anastomotic biliary stricture, primary graft nonfunction, decreased graft survival and even rejection may be attributed to PRI (10-12). Many studies are targeted towards the damage mechanism and production of preservation solutions to lessen the injury (13-15).

Subtle histopathologic changes seen in time-zero liver biopsies are harbingers of obvious damage in proceeding days (16). Prediction of graft survival, based on histopathologic and laboratory findings of early post-transplantation period, is of interest $(11,17)$. A recent study has

Copyright ( ) 2015, Kowsar Corp. This is an open-access article distributed under the terms of the Creative Commons Attribution-NonCommercial 4.0 International License (http://creativecommons.org/licenses/by-nc/4.0/) which permits copy and redistribute the material just in noncommercial usages, provided the original work is properly cited. 
shown that severe PRI in time-zero liver biopsy is an independent determinant of one-year graft survival (18).

\section{Objectives}

In the present study, we correlated short-term posttransplantation laboratory findings and characteristics of donor and recipients with certain histopathologic signs of PRI in time-zero liver biopsies. The aim of our study was to identify correlation of major histopathologic changes related to PRI with the alterations in liver tests in the early post-transplantation period.

\section{Materials and Methods}

This study was performed on 94 consecutive cases of orthotopic liver transplantations referred to Imam Khomeini Hospital Complex affiliated to Tehran University of Medical Sciences (TUMS) between April 2013 and August 2014. Livers were harvested from brain-dead donors and the organs were kept in University of Wisconsin solution before transplantation. Reperfusion was done on portal flow after flush of $1000 \mathrm{~mL}$ room temperature Ringer's lactate and $300 \mathrm{~mL}$ portal blood. Demographic, laboratory, radiologic findings and other clinical data of both recipients and donors were recorded.

In our institution, the time-zero liver needle biopsy is routinely taken from all transplanted livers after completion of vascular and biliary anastomoses in the operating room. Protocol, Tru-Cut, time-zero liver needle biopsies were placed in $10 \%$ buffered formalin solution and sent to pathology department. All biopsy specimens were reviewed by two pathologists who were blind to the patients' identity and follow-up information. In the first step, the histopathologic changes were evaluated separately by the observers. In case of any discrepancy, the slides were reevaluated to resolve any mismatch. Therefore, the histopathologic findings for each case were recorded based on mutual agreement. The samples with at least four portal tracts were considered adequate. Histopathologic features attributed to ischemia/reperfusion injury and macro- or micro-vesicular steatosis were evaluated on Hematoxylin and Eosin (H \& E)-stained slides. Hepatocyte ballooning degeneration, steatosis, bilirubinostasis, presence of apoptotic bodies, bile plugs, neutrophilic infiltration and central confluent necrosis were evaluated and graded according to the following criteria:

Ballooning degeneration was defined as hepatocellular swelling, vacuolization of the cytoplasm and cytoplasmic clumping of filamentous eosinophilic material. The ballooning degeneration was graded as absent, few or many using the same criteria as proposed for nonalcoholic steatohepatitis (19).

Presence of multiple tiny cytoplasmic droplets, smaller than the nucleus of hepatocyte without displacement of the nucleus was defined as micro-vesicular steatosis, and a single large droplet displacing the nucleus as steatosis of macrovesicular type. The percentage of each type of steatosis was recorded. Macrovesicular steatosis was divided into the following categories: 0\% - 4\% (negligible), 5\% - 33\% (mild), 34\% - 66\% (moderate), more than 67\% (severe). Our suggested categories of microvesicular steatosis included 0\% (negative), $1 \%$ - 10\% (mild), more than 11\% (marked).

Bilirubinostasis (hepatocellular cholestasis) was considered hepatocyte discolorations by brown pigmented material indicative of retention of biliary constituents within hepatocytes. This pigmentation is mostly perivenular with extension towards the portal tracts in more severe instances. Presence or absence of bilirubinostasis and the involved acinar zones were recorded.

Apoptotic cells were identified by cytoplasmic shrinkage and eosinophilia, chromatin condensation and karyorrhexis. These apoptotic cells were counted in some $10 \times$ objective fields and mean value was obtained. The presence of more than one apoptotic cell per $10 \times$ objective field was defined as "many" in our grading scheme. Less than this amount was considered "few".

Presence of bile plug, defined as retention of bile in a distended bile canaliculus, is reported without further stratification. Neutrophilic infiltration within the liver tissue also was graded. A neutrophilic aggregate was defined as a cluster of at least three neutrophils. Existence of more than one such aggregates in each 10x objective field was scored as "many aggregates".

The cases were categorized based on presence or absence of perivenular (acinar zone 3) confluent necrosis. The causes of laboratory alterations including rejection (pathologically- or clinically-proven), hepatic artery thrombosis (radiologically-proven) and biliary complications were evaluated.

Hepatocellular and cholestatic damages are two main types of liver injury. In the former, we usually observe a rise in transaminases and lactate dehydrogenase (LDH), on the other hand, elevated blood levels of bilirubin and alkaline phosphatase are seen in the latter. The results of serum transaminases, alkaline phosphatase, bilirubin and lactate dehydrogenase were recorded on days 1, 3, 5, 7, 9, 11, 13 and 15 post-transplantation.

\subsection{Statistical Considerations}

The statistical studies were performed using SPSS 16.0 software. As the distribution of results for most variables was not normal, nonparametric statistics were used in this study. The numerical data were summarized as median and range. The histopathologic features of the timezero liver needle biopsy were compared with the clinical and laboratory findings of both donors and recipients using nonparametric tests including Mann-Whitney U and Kruskal-Wallis H statistics. A P value $<0.05$ was considered statistically significant.

\subsection{Ethical Issues}

No additional procedure or expenses were imposed on patients, and their identities were not disclosed. Moreover, this study was approved by the TUMS ethics committee. 


\section{Results}

From a total of 94 liver transplantation cases, 14, 4 and 3 cases were diagnosed with rejection, hepatic artery thrombosis and primary nonfunction, respectively. No biliary complication was found in the cases. No specific causes were diagnosed in the remaining 73 cases and their clinical courses progressed uneventfully.

Demographic, laboratory findings, days of stay in Intensive Care Unit (ICU) and ischemic times (cold and warm) of donors are summarized in Table 1. Male-female ratio of donors was 3.3.

The median age of recipients was 45 (ranges 15 to 60) years. Male-female ratio was 1.2. Hepatitis B infection and autoimmune hepatitis were the leading causes of cirrhosis, followed by cryptogenic cirrhosis. The histopathologic findings were presumably related to PRI and the degree of steatosis was compared to numerical and categorical variables (including liver tests, demography, and etiology of cirrhosis) of donors and recipients as follow:

A) Numerical variables: a. Neutrophilic infiltration showed a significant correlation with the following variables: total bilirubin of days 7 and 15 post-transplantation ( $\mathrm{P}=0.014$ and 0.03 , respectively) and AST of day 15 posttransplantation $(\mathrm{P}=0.046)$. b. Apoptotic cells revealed a significant correlation with ALT of day 1 post-transplantation $(\mathrm{P}=0.048)$, AST $(\mathrm{P}=0.04)$ and ALT $(0.03)$ of day 5 post-transplantation and cold ischemic time (0.004). c. Central necrosis demonstrated a significant correlation with total bilirubin (mean of 2.12 for cases without central necrosis and 3.00 in cases with central necrosis, $\mathrm{P}=0.012$ ) of day 3 post-transplantation and donors' serum sodium level (mean of 149.72 for cases without central necrosis and 141.46 in cases with central necrosis, $\mathrm{P}=0.011$ ). d. Macrovesicular steatosis categories (Table 2) show a significant correlation with ALT $(\mathrm{P}=0.02)$, LDH $(\mathrm{P}=0.04)$, total bilirubin $(\mathrm{P}=0.046)$ of day 1 post-transplantation, total bilirubin of days 3, 7, 9 and 11 post-transplantation $(P=0.04,0.02$, 0.002 and 0.03 , respectively), weight $(\mathrm{P}=0.02)$ and BMI of the donors $(\mathrm{P}=0.02)$. e. Microvesicular steatosis categories revealed a significant correlation with AST of day 5 posttransplantation $(\mathrm{P}=0.013)$, the days of ICU stay in donors $(P=0.009)$ and warm ischemic time $(P=0.047)$. $\mathrm{f}$. Amount and site of ballooning degeneration, bilirubinostasis, portal congestion and bile plug showed no significant correlation with any of the numerical variables.

B) Categorical variables: Cause of death, cause of hepatic failure and sex of donors showed no significant correlation with the histopathologic findings. Only sex of recipients was correlated with severity of apoptosis in liver biopsies $(\mathrm{P}=0.013)$, i.e. apoptosis was more severe in male recipients.

Considering the aforementioned results, the categories for neutrophilic infiltration and apoptotic cells were simplified and statistical studies repeated in the new categories as follows: Neutrophilic infiltration was categorized based on presence or absence of neutrophilic aggregates. This new categorization showed a significant correlation with the following variables: LDH and total bilirubin of day 1 post-transplantation, $(\mathrm{P}=0.02$ and 0.03$)$, and total bilirubin of days 5 and 7 post-transplantation $(P=0.013$ and 0.015) (Table 3).

\begin{tabular}{lc}
\hline Table 1. Donors' Characteristics ${ }^{\text {a }}$ & \\
\hline Variables & Median (Range) \\
\hline Age, $\mathbf{y}$ & $31.5(12-64)$ \\
Weight, kg & $73.2(45-95)$ \\
BMI, kg/m² & $24.6(19-35)$ \\
AST, U/L & $41(8-460)$ \\
ALT, U/L & $36(7-263)$ \\
Na, mEq/L & $146.5(127-266)$ \\
Height, cm & $171.4(150-190)$ \\
ICU stay, d & $4(2-17)$ \\
Cold Ischemia, min & $274.0(22-724)$ \\
Warm Ischemia, min & $38.5(22-61)$ \\
\hline a Abbreviations: ALT, alanine aminotransferase; AST, aspartate \\
aminotransferase; BMI, body mass index; ICU, intensive care unit; and \\
Na, sodium.
\end{tabular}

Table 2. Statistically Significant Laboratory Changes Associated With Macrovesicular Steatosis in Time-Zero Liver Needle Biopsy

\begin{tabular}{|c|c|c|c|c|c|c|c|c|c|}
\hline \multirow{2}{*}{$\begin{array}{l}\text { Macrovesicular } \\
\text { Steatosis Categor }\end{array}$} & \multirow[t]{2}{*}{ ALT-1 $^{a}$} & \multirow[t]{2}{*}{ LDH-1 $^{b}$} & \multirow[t]{2}{*}{ T-Bil-1 ${ }^{\mathrm{C}}$} & \multirow[t]{2}{*}{ T-Bil-3 ${ }^{\mathrm{d}}$} & \multirow[t]{2}{*}{ T-Bil-7 ${ }^{\mathrm{e}}$} & \multirow[t]{2}{*}{ T-Bil-9 ${ }^{f}$} & \multirow[t]{2}{*}{ T-Bil-11 ${ }^{g}$} & \multicolumn{2}{|c|}{ Donor } \\
\hline & & & & & & & & Weight, kg & BMI, $\mathrm{kg} / \mathrm{m}^{2}$ \\
\hline 0 - 4 & $519.2 \pm 832.24$ & $1287.09 \pm 1622.43$ & $4.38 \pm 5.15$ & $2.14 \pm 2.23$ & $1.59 \pm 1.17$ & $1.32 \pm 0.86$ & $1.39 \pm 0.86$ & $72.19 \pm 10.08$ & $24.6 \pm 2.61$ \\
\hline $5-30$ & $1074.71 \pm 1277.00$ & $1936.71 \pm 1118.18$ & $9.00 \pm 5.86$ & $3.21 \pm 2.04$ & $3.46 \pm 3.95$ & $3.37 \pm 3.25$ & $2.62 \pm 1.63$ & $82.71 \pm 10.32$ & $27.86 \pm 3.55$ \\
\hline Pvalue & 0.02 & 0.04 & 0.046 & 0.04 & 0.02 & 0.002 & 0.03 & 0.02 & 0.02 \\
\hline \multicolumn{10}{|c|}{$\begin{array}{l}\text { a } \text { LDH of day } 1 \text { post-transplantation, U/L (Mean } \pm \text { Standard deviation). } \\
\text { b Total bilirubin of day } 1 \text { post-transplantation, } \mathrm{mg} / \mathrm{dL} \text { (Mean } \pm \text { Standard deviation). } \\
\text { c ALT of day1 post-transplantation, U/L (Mean } \pm \text { Standard deviation). } \\
\text { d AST of day } 1 \text { post-transplantation, U/L (Mean } \pm \text { Standard deviation). } \\
\text { e Total bilirubin of day } 5 \text { post-transplantation, } \mathrm{mg} / \mathrm{dL} \text { (Mean } \pm \text { Standard deviation). } \\
\mathrm{f} \text { ALT of day } 5 \text { post-transplantation, U/L (Mean } \pm \text { Standard deviation). } \\
\text { g AST of day } 5 \text { post-transplantation, U/L (Mean } \pm \text { Standard deviation). } \\
\text { h Total bilirubin of day } 7 \text { post-transplantation, } \mathrm{mg} / \mathrm{dL} \text { (Mean } \pm \text { Standard deviation). } \\
\text { i ALT of day } 7 \text { post-transplantation, U/L (Mean } \pm \text { Standard deviation). } \\
\text { j AST of day } 7 \text { post-transplantation, U/L (Mean } \pm \text { Standard deviation). } \\
\text { k Not significant. }\end{array}$} \\
\hline
\end{tabular}


Shahbazi $N$ et al.

Table 3. Correlation of Presence of Neutrophilic Aggregates in Time-Zero Liver Biopsies With Results of Liver Laboratory Tests

\begin{tabular}{|c|c|c|c|c|c|c|c|c|c|c|}
\hline $\begin{array}{l}\text { Interstitial } \\
\text { Neutrophils }\end{array}$ & LDH-1 $^{a}$ & T-Bil-1 b & ALT-1 $^{C}$ & AST-1 $^{d}$ & T-Bil-5 ${ }^{\mathrm{e}}$ & ALT-5 ${ }^{\mathrm{f}}$ & AST-5 $\mathrm{g}$ & T-Bil-7 h & ALT-7 $^{\mathrm{i}}$ & ${\text { AST }-7^{j}}^{j}$ \\
\hline No aggregate & $996.34 \pm 920.90$ & $4.47 \pm 6.59$ & $532.84 \pm 974.71$ & $481,00 \pm 415,70$ & $1.57 \pm 1.44$ & $196,24 \pm 268,36$ & $54.97 \pm 47,07$ & $1.38 \pm 1.07$ & $7148.29 \pm 151,69$ & $57,60 \pm 42,06$ \\
\hline $\begin{array}{l}\text { Aggregate } \\
\text { present }\end{array}$ & $1715.64 \pm 1993.27$ & $5.26 \pm 3.78$ & $816.81 \pm 893.45$ & $1046,98 \pm 806,00$ & $1.90 \pm 1.11$ & $184,24 \pm 183.94$ & $55,94 \pm 30,56$ & $2.18 \pm 2.15$ & $148,24 \pm 140,23$ & $69.81 \pm 83.72$ \\
\hline value & 0.02 & 0.03 & $0.20^{k}$ & $0.08^{k}$ & 0.013 & $0.45^{\mathrm{k}}$ & $0.31^{\mathrm{k}}$ & 0.015 & $0.52^{\mathrm{k}}$ & $0.46^{k}$ \\
\hline
\end{tabular}

a ALT of day 1 post-transplantation, $\mathrm{U} / \mathrm{L}$ (Mean \pm Standard deviation).

$\mathrm{b}$ Total bilirubin of day 1 post-transplantation, $\mathrm{mg} / \mathrm{dL}$ (Mean \pm Standard deviation).

c ALT of day 3 post-transplantation, $\mathrm{U} / \mathrm{L}($ Mean \pm Standard deviation).

$\mathrm{d}$ Total bilirubin of day 3 post-transplantation, $\mathrm{mg} / \mathrm{dL}$ (Mean \pm Standard deviation).

e AST of day 5 post-transplantation, $\mathrm{U} / \mathrm{L}$ (Mean \pm Standard deviation).

$\mathrm{f}$ ALT of day 5 post-transplantation, $\mathrm{U} / \mathrm{L}$ (Mean \pm Standard deviation).

$\mathrm{g}$ Total bilirubin of day 5 post-transplantation, $\mathrm{mg} / \mathrm{dL}$ (Mean \pm Standard deviation).

$\mathrm{h}$ ALT of day 5 post-transplantation, $\mathrm{U} / \mathrm{L}$ (Mean \pm Standard deviation).

i Total bilirubin of day 9 post-transplantation, $\mathrm{mg} / \mathrm{dL}$ (Mean \pm Standard deviation).

j Not significant.

\begin{tabular}{|c|c|c|c|c|c|c|c|c|c|}
\hline Apoptotic Cells & ALT- ${ }^{a}$ & T-Bil-1 ${ }^{b}$ & ALT-3 ${ }^{C}$ & T-Bil-3 ${ }^{d}$ & AST-5 ${ }^{\mathrm{e}}$ & ALT- $5^{\mathrm{f}}$ & T-Bil-5 $g$ & ALT-9 $\mathrm{h}$ & T-Bil-9 ${ }^{\mathrm{i}}$ \\
\hline Absent & $318.91 \pm 247.27$ & $4.68 \pm 3.84$ & $263.70 \pm 439.24$ & $2.07 \pm 1.66$ & $45.18 \pm 29.07$ & $145.85 \pm 197.78$ & $1.56 \pm 1.16$ & $101.93 \pm 90.95$ & $1.60 \pm 1.77$ \\
\hline Present & $822.56 \pm 1178.11$ & $5.04 \pm 6.58$ & $452.62 \pm 666890$ & $2.43 \pm 2,65$ & $65.70 \pm 46.14$ & $233.50 \pm 251.14$ & $1.91 \pm 1.40$ & $146.62 \pm 111.53$ & $1.46 \pm 0.95$ \\
\hline Pvalue & 0.02 & $0.56^{j}$ & 0.04 & $0.59^{j}$ & 0.011 & 0.010 & $0.22^{j}$ & 0.03 & $0.64^{j}$ \\
\hline
\end{tabular}

${ }^{\mathrm{a}}$ ALT of day 1 post-transplantation, $\mathrm{U} / \mathrm{L}$ (Mean \pm Standard deviation).

$\mathrm{b}$ LDH of day 1 post-transplantation, $\mathrm{U} / \mathrm{L}$ (Mean \pm Standard deviation).

c Total bilirubin of day 1 post-transplantation, $\mathrm{mg} / \mathrm{dL}$ (Mean \pm Standard deviation).

$\mathrm{d}$ Total bilirubin of day 3 post-transplantation, $\mathrm{mg} / \mathrm{dL}$ (Mean \pm Standard deviation).

e Total bilirubin of day 7 post-transplantation, $\mathrm{mg} / \mathrm{dL}$ (Mean \pm Standard deviation).

${ }^{\mathrm{f}}$ Total bilirubin of day 9 post-transplantation, $\mathrm{mg} / \mathrm{dL}$ (Mean \pm Standard deviation).

$\mathrm{g}$ Total bilirubin of day 11 post-transplantation, $\mathrm{mg} / \mathrm{dL}$ (Mean \pm Standard deviation).

Biopsies were categorized based on presence or absence of apoptotic cells and this new grouping was correlated with ALT of days 1,3,5 and 9 post-transplantation $(\mathrm{P}=0.02$, $0.04,0.01$ and 0.03 ) and AST of day 5 post-transplantation $(\mathrm{P}=0.011)$ (Table 4).

\section{Discussion}

The preservation/reperfusion injury is common in liver transplantation, particularly when the organ is procured from brain-dead donors in comparison to living donors (20). There are different causes for abnormal liver function tests in early post-transplantation period including PRI, hyperacute or acute rejection, hepatic artery or biliary tree complications. These events can lead to primary graft failure in their severe states. Hepatic artery thrombosis and biliary tract complication can be diagnosed by imaging modalities and treated accordingly. Considering different management of PRI and rejection, their distinction is of therapeutic and prognostic importance (21). However, liver biopsy, an invasive procedure, is required to definitely differentiate these two entities. To predict abnormal laboratory findings that can be attributed to PRI, we evaluated the histopathologic findings of timezero liver biopsies.

To determine the most important histopathologic changes attributed to PRI and their relation with early post-transplantation laboratory findings, we excluded other potential causes of altered laboratory findings, namely hyperacute/acute rejection, hepatic and biliary complications and primary nonfunction. Therefore, we assume that all the laboratory alterations in our cases are caused by non-graft threatening PRI.

Time-zero liver needle biopsy is indicative of histopathologic changes attributed to donor including steatosis, chronic hepatitis and fibrosis, as well as changes imposed on the allograft because of cold and warm ischemia during procurement and transplantation of the organ. In this study, the histopathologic findings of timezero liver needle biopsies were scrutinized and different parameters were graded. We compared these histopathologic findings with the liver function tests to identify the most important histopathologic changes that can reflect abnormal liver function tests. Of different parameters that were evaluated macrovesicular steatosis, apoptotic bodies and neutrophilic aggregates seem to be the most important histopathologic changes. To grade the severity of PRI in time-zero liver needle biopsies, Ali et al. also used neutrophilic infiltrate, apoptosis and hepatocytes dropout in a recent study (18).

Other studies have shown that warm ischemia is associated with hepatocellular injury, and cold ischemia can result in sinusoidal endothelial and bile duct epithelial 
cell damage $(1,2,7)$. In our study, we found that presence and density of apoptotic bodies were correlated with increase in transaminases in early post-transplantation period. These changes were in favor of hepatocellular type of liver injury caused by warm ischemia. Conversely, neutrophilic aggregates were mainly associated with early increase in total bilirubin blood level. Identification of bile duct epithelial and sinusoidal endothelial cell damage (resulting from cold ischemia) is beyond the scope of light microscopic evaluation particularly in time-zero liver biopsies. Nevertheless, neutrophilic aggregates which are accompanied by endothelial cell damage are easily identified on light microscopy. Therefore, in time-zero biopsies, we can consider apoptotic bodies and neutrophilic aggregates as surrogate markers of damage caused by warm and cold ischemia, respectively.

Macrovesicular steatosis makes hepatocytes more susceptible to both warm and cold ischemia. This susceptibility is probably caused by reduced glycogen stores of hepatocytes. Along similar lines, we showed macrovesicular steatosis is associated with mixed hepatocellular and cholestatic types of liver test alterations with increase in serum ALT, LDH and bilirubin.

If any laboratory abnormality is identified in a patient with time-zero liver biopsy findings indicative of no or mild PRI, the power of suspicion for alternative etiologies such as rejection or surgical complications should be increased. Based on the present study, considering causes other than PRI seems reasonable if there is any discrepancy between time-zero PRI histopathologic changes and pattern of early liver laboratory alterations. For example, if cholestatic pattern of laboratory abnormalities is identified in a patient with prominent apoptotic bodies in time-zero biopsy, PRI may be less incriminated as the etiology of laboratory changes.

Significantly more apoptotic bodies were identified in male versus female recipients. Although the exposure time of organ with recipients' sex hormones is short, the observed change may be attributed to apoptosis suppression as a result of estrogen. In this regard, an experimental study on rats has shown the effect of estrogen on suppression of apoptosis in neuronal cells (22). Moreover, Marino et al. have shown that male to female, in comparison to female to male, liver transplantations have a better two-year survival probably due to sex hormone linkage to human leukocyte antigen (HLA) expression (23).

We observed a statistically significant association between central confluent necrosis and increased total bilirubin. Also, a correlation was identified between microvesicular change and warm ischemic time, length of ICU admission of donors and post-transplantation rise in the AST level. To show the clinical significance of these findings, further studies are required.

Of different histopathologic findings observed in timezero liver needle biopsy, apoptotic bodies, neutrophilic aggregates and macrovesicular steatosis are the main changes that can reflect laboratory alterations caused by PRI in transplanted livers. Additionally, these three changes are associated with hepatocellular, cholestatic and mixed alterations of liver function tests, respectively.

\section{Acknowledgements}

The authors wish to thank the Iranian tissue bank research center for the assistance in laboratory affairs. Dr. Niknejad and Dr. Hamrahi have also helped us in data collection. This study was financially supported by vicechancellor for research, Tehran University of Medical Sciences (grant no 92-01-30-21718).

\section{Authors' Contributions}

Study concept and design: Ali Jafarian, Mohsen Nasiri Toosi, Farid Azmoudeh-Ardalan, and Narges Shahbazi. Acquisition of data: Monavar Talebian Moghadam, Narges Shahbazi, Farid Azmoudeh-Ardalan, and Hayedeh Haeri. Analysis and interpretation of data: Farid Azmoudeh-Ardalan, and Narges Shahbazi. Drafting of the manuscript: Narges Shahbazi, Farid Azmoudeh-Ardalan, Mohsen Nasiri Toosi, and Ali Jafarian. Critical revision of the manuscript for important intellectual content: Mohsen Nasiri Toosi, Reza Shahsiah, Narges Shahbazi, and Hayedeh Haeri. Statistical analysis: Farid Azmoudeh-Ardalan, Narges Shahbazi. Administrative, technical, and material support: Sedigheh S. Poursaleh, Farid Azmoudeh-Ardalan, and Ali Jafarian. Study supervision: Hayedeh Haeri, Ali Jafarian, and Reza Shahsiah.

\section{Funding/Support}

This study was financially supported by vice-chancellor for research, Tehran University of Medical Sciences (grant no 92-01-30-21718).

\section{References}

1. Kohli V, Selzner M, Madden JF, Bentley RC, Clavien PA. Endothelial cell and hepatocyte deaths occur by apoptosis after ischemia-reperfusion injury in the rat liver. Transplantation. 1999;67(8):1099105.

2. Natori S, Selzner M, Valentino KL, Fritz LC, Srinivasan A, Clavien PA, et al. Apoptosis of sinusoidal endothelial cells occurs during liver preservation injury by a caspase-dependent mechanism. Transplantation. 1999;68(1):89-96.

3. Lemasters JJ, Thurman RG. Reperfusion injury after liver preservation for transplantation. Annu Rev Pharmacol Toxicol. 1997;37:327-38.

4. Bilzer M, Gerbes AL. Preservation injury of the liver: mechanisms and novel therapeutic strategies. J Hepatol. 2000;32(3):508-15.

5. Montalvo-Jave EE, Escalante-Tattersfield T, Ortega-Salgado JA Pina E, Geller DA. Factors in the pathophysiology of the liver ischemia-reperfusion injury. J Surg Res. 2008;147(1):153-9.

6. Serracino-Inglott F, Habib NA, Mathie RT. Hepatic ischemia-reperfusion injury. Am J Surg. 2001;181(2):160-6.

7. Kupiec-Weglinski JW, Busuttil RW. Ischemia and reperfusion injury in liver transplantation. Transplant Proc. 2005;37(4):1653-6.

8. Kukan M, Haddad PS. Role of hepatocytes and bile duct cells in preservation-reperfusion injury of liver grafts. Liver Transpl. 2001;7(5):381-400.

9. Hickman PE, Potter JM, Pesce AJ. Clinical chemistry and postliver-transplant monitoring. Clin Chem.1997;43(8 Pt 2):1546-54.

10. Pirenne J, Van Gelder F, Coosemans W, Aerts R, Gunson B, Koshiba 
T, et al. Type of donor aortic preservation solution and not cold ischemia time is a major determinant of biliary strictures after liver transplantation. Liver Transpl. 2001;7(6):540-5.

11. Kakizoe S, Yanaga K, Starzl TE, Demetris AJ. Evaluation of protocol before transplantation and after reperfusion biopsies from human orthotopic liver allografts: considerations of preservation and early immunological injury. Hepatology.1990;11(6):932-41.

12. Howard TK, Klintmalm GB, Cofer JB, Husberg BS, Goldstein $\mathrm{RM}$, Gonwa TA. The influence of preservation injury on rejection in the hepatic transplant recipient. Transplantation. 1990; 49(1):103-7.

13. Schon MR, Kollmar O, Wolf S, Schrem H, Matthes M, Akkoc N, et al. Liver transplantation after organ preservation with normothermic extracorporeal perfusion. Ann Surg. 2001;233(1):114-23.

14. Yagnik GP, Takahashi Y, Tsoulfas G, Reid K, Murase N, Geller DA Blockade of the L-arginine/NO synthase pathway worsens hepatic apoptosis and liver transplant preservation injury. Hepatology. 2002;36(3):573-81.

15. Ploeg RJ, D'Alessandro AM, Knechtle SJ, Stegall MD, Pirsch JD, Hoffmann RM, et al. Risk factors for primary dysfunction after liver transplantation--a multivariate analysis. Transplantation. 1993;55(4):807-13.

16. Neil DA, Hubscher SG. Are parenchymal changes in early posttransplant biopsies related to preservation-reperfusion injury or rejection? Transplantation. 2001;71(11):1566-72

17. Pokorny H, Gruenberger T, Soliman T, Rockenschaub S, Langle F, Steininger R. Organ survival after primary dysfunction of liver grafts in clinical orthotopic liver transplantation. Transpl Int. 2000;13 Suppl 1:S154-7.

18. Ali JM, Davies SE, Brais RJ, Randle LV, Klinck JR, Allison ME, et al. Analysis of ischemia/reperfusion injury in time-zero biopsies predicts liver allograft outcomes. Liver Transpl. 2015;21(4):487-99.

19. Brunt EM, Janney CG, Di Bisceglie AM, Neuschwander-Tetri BA Bacon BR. Nonalcoholic steatohepatitis: a proposal for grading and staging the histological lesions. Am J Gastroenterol. 1999;94(9):2467-74.

20. Weiss S, Kotsch K, Francuski M, Reutzel-Selke A, Mantouvalou $\mathrm{L}, \mathrm{Klemz} \mathrm{R}$, et al. Brain death activates donor organs and is associated with a worse I/R injury after liver transplantation. Am J Transplant. 2007;7(6):1584-93.

21. Fondevila C, Busuttil RW, Kupiec-Weglinski JW. Hepatic ischemia/ reperfusion injury--a fresh look. Exp Mol Pathol. 2003;74(2):86-93.

22. Arai Y, Sekine Y, Murakami S. Estrogen and apoptosis in the developing sexually dimorphic preoptic area in female rats. Neurosci Res. 1996;25(4):403-7.

23. Marino IR, Doyle HR, Aldrighetti L, Doria C, McMichael J, Gayowski T, et al. Effect of donor age and sex on the outcome of liver transplantation. Hepatology. 1995;22(6):1754-62. 